\title{
Dynamics of Proteins by Thermal Decay of Free Radicals Induced by Ultraviolet Irradiation
}

\author{
Rasim B. Aslanov, ${ }^{1}$ Leman M. Dashdemirova, ${ }^{1}$ Oktay Z. Alekperov, ${ }^{2}$ \\ Azad R. Abdurahimov, ${ }^{1}$ and Oktay K. Gasymov $\mathbb{D}^{1}$ \\ ${ }^{1}$ Institute of Biophysics, Azerbaijan National Academy of Sciences, 117 Z. Khalilov, Baku 1141, Azerbaijan \\ ${ }^{2}$ Institute of Physics, Azerbaijan National Academy of Sciences, 131G. Javid, Baku 1143, Azerbaijan \\ Correspondence should be addressed to Oktay K. Gasymov; oktaygasimov@gmail.com
}

Received 13 March 2018; Revised 20 May 2018; Accepted 29 May 2018; Published 28 June 2018

Academic Editor: Feride Severcan

Copyright ( 2018 Rasim B. Aslanov et al. This is an open access article distributed under the Creative Commons Attribution License, which permits unrestricted use, distribution, and reproduction in any medium, provided the original work is properly cited.

\begin{abstract}
The relationship between structure, dynamics, and function of biomolecules is a fundamental interest of biophysics. Protein dynamics drastically vary in temporal and spatial scales. The function of a particular protein determines the significance of a distinct type of dynamics. Here, we investigate the influence of hydration water on the dynamics of a protein called silk fibroin. Particular interest is to investigate the protein dynamics using thermal decay of the free radicals induced by ultraviolet irradiation. The full decay of the free radicals occurs at very wide temperature region $(120 \mathrm{~K}-340 \mathrm{~K})$. Three distinct regions with transition points of $\sim 135 \mathrm{~K}, 205 \mathrm{~K}$, and $279 \mathrm{~K}$ are apparent in the thermal decay curves of hydrated fibroin samples. The first transition $(\sim 135 \mathrm{~K})$ that leads $2-6 \%$ increase of total spins was observed only in the decay curves of fibroin submerged in $40 \%$ and $50 \%$ glycerol. The second transition $(\sim 205 \mathrm{~K})$ was invariant for all samples, hydrated and dry fibroins. The third transition of $279 \mathrm{~K}$ common for all hydrated fibroin samples was shifted about $84 \mathrm{~K}$ to a higher temperature of $363 \mathrm{~K}$ in dry fibroin. The thermal transitions at $205 \mathrm{~K}$ and $279 \mathrm{~K}$ are weakly and strongly, respectively, coupled to water molecules. Nature of the free radicals participated in these transitions was identified. The significance of the findings for protein dynamics is discussed.
\end{abstract}

\section{Introduction}

Protein structure and dynamics are essential to understand their functions. The dynamics of proteins cover extremely wide spatial and temporal scales and largely determined by the rugged energy landscape and fluctuations $[1,2]$. The basic concept of the protein energy landscape was formulated by studies of the temperature dependence of protein structure and dynamics [3]. The physical state of a protein, as well as a type of the studied dynamics, determines the relevant temperature interval. The temperature dependence of protein dynamics has been studied by fundamental methods such as nuclear magnetic resonance (NMR), X-ray diffraction, neutron scattering, electron paramagnetic resonance (EPR), and fluorescence. Each method has their own timescale to measure protein dynamics.

The stability of the free radicals induced by the external physical factors, such as ultraviolet (UV) and $\gamma$-irradiations, in proteins is very temperature dependent. However, much less attention is paid to study the protein dynamics using the free radicals of proteins as a "reporter group." As in other dynamic modes, the dynamics of the free radicals and, therefore, their reactivity should also depend on protein structure and hydration states.

Free radical formation has been observed in many biological processes, for example, oxidative stress, and may damage the cell components [4]. The propagation of free radicals in cell components and their reactions with antioxidants have the utmost importance for a living system. Proteins as one of the essential components in intracellular as well as in extracellular environment perform various functions that are fundamental to support the living system. Free radicals in proteins may be generated by direct and indirect mechanisms. Regardless of details of free radical formation mechanism, various free radical reactions determine response and fate of the cell.

The objective of the paper is to study the influence of structure and hydration of the proteins on decay 
(recombination and disproportionation) properties of the free radicals induced by UV irradiation of the protein. Silk fibroin is taken as paradigm protein for a family of fibrous proteins.

Silk fibroin is an excellent biomaterial that has been widely applied to different fields [5-11]. Fibroin also shows excellent biocompatibility properties [12]. Compared with many other biomolecules, fibroin with highly organized beta-sheet structure shows slow degradation in in vitro as well as in vivo conditions [13]. Silk fibroin consists of two chains, light and heavy chains with molecular weights of 25 and $325 \mathrm{kDa}$, respectively [14]. A heavy chain is a major structural component of fibroin and largely composed of repeats of (Gly-Ala-Gly-Ala-Gly-Ser) ${ }_{n}$, which are responsible for beta-sheet formation. About $80-85 \%$ of fibroin is constituted from Gly, Ala, and Ser. Silk fibroin is considered as semicrystalline material, which consists of crystalline and amorphous parts [15]. This peculiar structural organization determines many properties of fibroins.

There are a number of published works dedicated to the study of UV irradiation on silk fibroin [16-20]. In all cases, similar EPR spectra of free radicals have been observed in the absence of oxygen. Good agreement exists on the formation of the initial free radicals by UV irradiation of silk fibroin. Yet, the nature of free radicals produced in subsequent reactions is ambiguous. Time dependence of decay reactions of the free radicals produced by UV irradiation of silk fibroin has been studied at various temperatures [17]. However, general temperature decay, the transition points of the reaction, and the nature of free radicals within decay kinetics were not considered. In this work, we aim to fill this gap.

By studying temperature-dependent decay of the free radicals induced by ultraviolet irradiation, we determined several transition temperatures. Investigation of the dry and hydrated forms of fibroin reveals the dynamics modes, which are differently coupled to water molecules. The origin of free radicals and their dynamics are discussed.

\section{Materials and Methods}

2.1. Sample Preparation. Fibroin fibers were obtained by degumming of the silk cocoons of Bombyx mori using a widely accepted method [21]. Briefly, silk cocoons were cut into small pieces and then boiled for $30 \mathrm{~min}$ in $0.02 \mathrm{M}$ $\mathrm{Na}_{2} \mathrm{CO}_{3}$. Degummed silk fibers were washed thoroughly with deionized water and then dried. In hydration experiments, fibroin fibers were incubated in pure water and various water/glycerol mixtures (40\% and 50\% v/v) for at least 24 hours. The investigated samples were kept submerged in the abovementioned solutions.

2.2. UV Irradiation. All samples were irradiated at $77 \mathrm{~K}$ with focusing light of ultra-high-pressure mercury lamp (500 watts) during 1 hour. The dry sample was flushed with argon for several minutes and kept in the noble gas environment. Dissolved gas in water/glycerol mixtures was removed by a freeze-thaw method. To eliminate uncertainty from UV dose dependence, all samples were irradiated to have a formation of the maximum free radicals (spin per gram). One hour of UV irradiation of fibroin fiber yields $95-100 \%$ of the maximum of free radicals. Before performing a free radical recombination experiment, each sample was examined to confirm that the samples have about the same EPR intensity. It makes sure that each sample had the same UV radiation dose. On the other hand, previously it has been shown that the decay rate of free radical recombination depends linearly on the initial concentration [22]. Therefore, the free radical recombination reactions normalized to the initial concentrations will yield the same reaction parameters.

2.3. EPR Spectroscopy. EPR spectra were recorded using Bruker ELEXSYS E580 spectrometer at X-band frequency with variable temperature accessory. Samples irradiated by ultraviolet (UV) at $77 \mathrm{~K}$ were kept 15 minutes at various temperatures and, then, immediately plunged to liquid nitrogen to stop heat-induced decay of the free radicals. The experiments were repeated three to five times. All spectra were recorded at $77 \mathrm{~K}$ that eliminates the requirement for a correction procedure that accounts for the temperature variation in calculations of free radical concentrations. Double integration of EPR spectra was performed for total spin quantitation in thermal reactions. EPR measurements were conducted using an incident microwave power of $0.47 \mathrm{~mW}$ and modulation amplitude of $2 \mathrm{G}$. Scan width was 200G. The hyperfine splitting constants and g-factors of the free radicals were determined using the software provided with the instrument. Double integrations of EPR spectra were performed using OriginLab software (OriginLab Corp., Northampton, MA). To minimize the error produced by a baseline selection of the spectrum, double integration was performed in two steps. After performing a single integration of the first derivative spectrum, the new baseline was created to yield a correct absorption band. Second integration was performed using the corrected baseline.

\section{Results and Discussion}

3.1. EPR Spectra of the Free Radicals Induced by UV Irradiation of Silk Fibroin. The temperature dependence of EPR spectra of the free radicals induced by UV irradiation of silk fibroin in both dry and hydrated forms is shown in Figure 1. EPR spectra display comparable spectral characteristics, doublet spectrum with $g=2.004$, and similar (details are given below) hyperfine splitting constant of $\sim 16 \mathrm{G}$. It is evident that hydration does not change the nature of the produced free radicals. The formation of free radicals in proteins occurs via ionization of aromatic residues, mostly Trp and Tyr [23]. In case of fibroin, mostly Tyr residues will be ionized. Excitation of silk fibroin at $275 \mathrm{~nm}$ produces fluorescence emission with a maximum at $305 \mathrm{~nm}$, which is specific for Tyr residues [19]. Because of low abundance of Trp residues in silk fibroin, the fluorescence emission from Trp is not visible at this excitation wavelength. The fluorescence emission of fibroin excited at $280 \mathrm{~nm}$ depends on the secondary structure of the protein [24]. However, the 


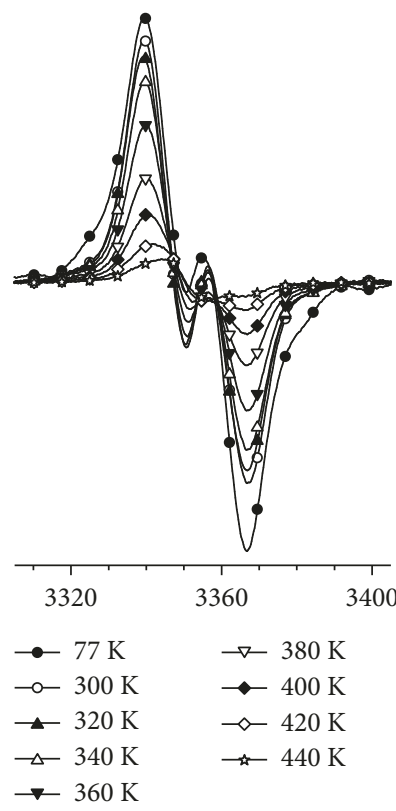

(a)

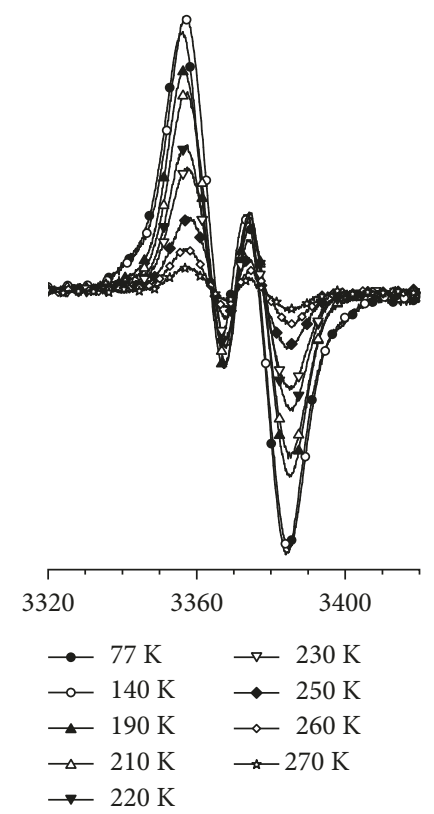

(b)
FIGURE 1: EPR spectra of the free radicals induced by UV irradiation of silk fibroin annealed at various temperatures. Fibroin in (a) dry and (b) hydrated forms in $50 \%$ glycerol solution. All spectra were taken at $77 \mathrm{~K}$.

fluorescence emission of fibroin is only related to Tyr or Trp. Therefore, UV irradiation of silk fibroin leads to the formation of excited states of Tyr and/or Trp. The excited state of the aromatic residues may undergo the radiative relaxation (fluorescence emission) or nonradiative relaxations, one of which is ionization. Ionization of Trp may occur from the nonrelaxed prefluorescent $S^{*}$ and relaxed fluorescent $S_{1}$ states [25]. In solution, ejected electrons from the aromatic residues yield solvated electrons, $e_{\mathrm{aq}}^{-}$. The cation radicals of the aromatic residues quickly deprotonate and produce the corresponding neutral radicals [23]:

$$
\begin{aligned}
\operatorname{Trp}+\mathrm{hv} & \longrightarrow \operatorname{Trp}^{\bullet+}+e_{\mathrm{aq}}^{-} \\
\operatorname{Trp}^{\bullet+}+\mathrm{hv} & \longrightarrow \operatorname{Trp}{ }^{\bullet}+\mathrm{H}^{+}
\end{aligned}
$$

The final products of photoionization of Tyr residues are similar to those of Trp, neutral $\mathrm{Tyr}-\mathrm{O}^{\bullet}$ radicals, and solvated electrons, $e_{\mathrm{aq}}^{-}$. Various reactions can occur with initially generated free radicals of aromatic residues. However, the most relevant for silk fibroin is free radicals localized in the polypeptide chain. The carbon atoms of the carbonyl groups of the polypeptide chain can capture the solvated electrons to yield ketyl radicals, also known as carbon radicals [23].

$$
e_{\mathrm{aq}}^{-}+-\mathrm{CONH}-\longrightarrow \mathrm{OH}^{-}+-\mathrm{C}^{\bullet}(\mathrm{OH}) \mathrm{NH}-
$$

The carbon radicals can propagate along the polypeptide chain and participate in disproportionation and recombination reactions. In dry proteins, electrons ejected from the aromatic residues can directly be captured by the carbon atoms of carbonyl groups, bypassing the solvated

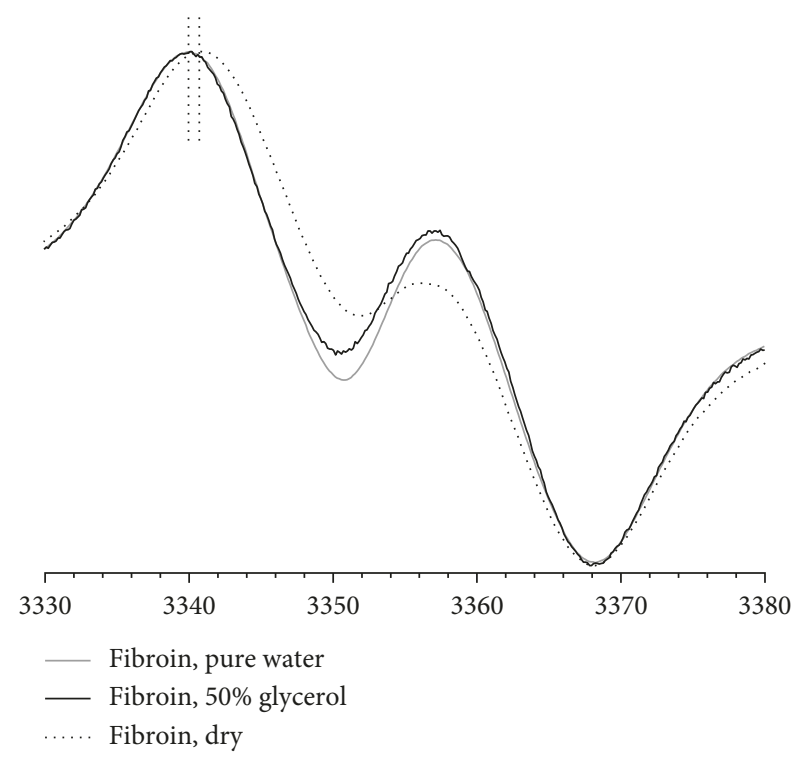

FIGURE 2: EPR spectra of free radicals induced by UV irradiation of silk fibroin in dry form and immersed in water and 50\% glycerol at $77 \mathrm{~K}$. For better visualization, both wings of the spectra were truncated.

state of electrons. Therefore, the nature of free radicals induced by UV irradiation and their respective EPR spectra expected to be very similar in the dry and hydrated forms of fibroin (Figure 1).

EPR spectra of the free radicals of fibroin in dry and hydrated forms are dominated by doublet lines that arise from the interaction of electron spin of the carbon atom with the nearest proton. Compared to the hydrated fibroins, one distinct feature is evident for the EPR spectra of dry fibroin. In dry forms, the resolutions of doublet lines are poor compared to those of hydrated forms. The small difference in hyperfine splitting constant is evident in EPR spectra of dry and hydrated samples (Figure 2). The hyperfine splitting constant for doublet EPR spectrum of dry fibroin (16.2 G) increased about $1 \mathrm{G}$ for hydrated fibroin samples.

The hyperfine splitting is the same for fibroin immersed in water and 50\% glycerol solutions. Data indicate that the free radicals responsible for the doublet spectra are solvated.

The singlet EPR spectrum is emerging at a higher temperature in the dry form of fibroin (Figure 1(a)) that is not apparent in hydrated forms (Figure 1(b)). In hydrated forms, the backbone dynamics is higher and free radical decay of the $\alpha$-carbon radicals is more complete at relatively lower temperature. The appearance of singlet EPR line at $440 \mathrm{~K}$ may indicate the beginning of carbonization process. The temperature-dependent decay of free radicals in the various forms of fibroin should reveal the peculiarities of the emerging dynamics in the protein.

It should be noted that electron-beam irradiation of silk fibroin produces the similar doublet EPR spectrum [26, 27]. Evidently, both electrons from the beam and electrons ejected from the aromatic residues are captured by the same group, the carbon atom of the carbonyl group. However, thermal decays of these radicals were not investigated. 


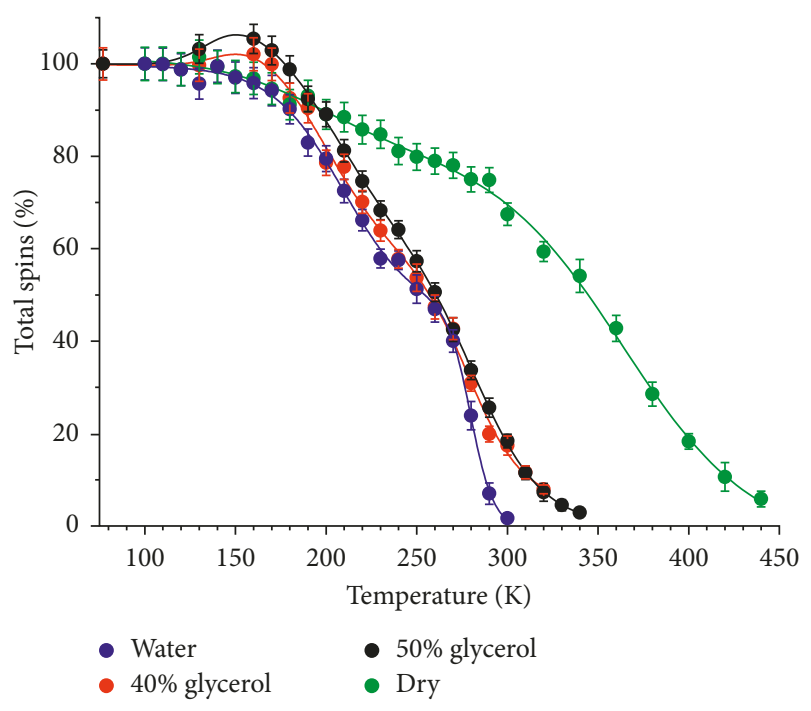

(a)

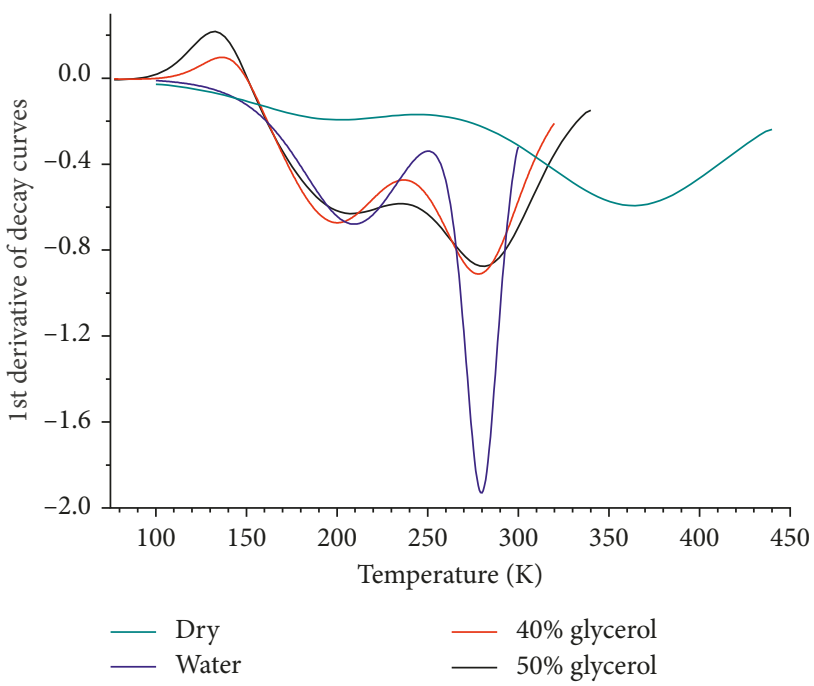

(b)

Figure 3: (a) The thermal decay curves of the free radicals induced by UV irradiation of silk fibroin in various hydrated and dry states and their respective first derivative curves. (b) Derivative curves were obtained using smooth lines connecting data points.

3.2. Thermal Decay of the Free Radicals Induced by UV Irradiation of Silk Fibroin. The thermal decay curves of the free radicals of silk fibroin in various hydrated and dry states are shown in Figure 3(a).

Fibroin samples immersed in $40 \%$ and $50 \%$ glycerol solutions show increased total number of spins in the temperature interval of $114 \mathrm{~K}-151 \mathrm{~K}$. In these samples, the solution around fibroin produces transparent glass upon freezing at $77 \mathrm{~K}$. Formation of free radicals at increased temperatures $(114 \mathrm{~K}-151 \mathrm{~K})$ can be explained by "cage effect." Upon UV irradiation of fibroin, some of the generated pairs of free radicals (free radicals always created in pairs) are trapped in the "cages." Due to a close proximity of the free radicals paired in the cage, the spectral lines become very broad due to spin-spin interactions. Therefore, the radical pairs trapped in these cages are invisible in EPR spectra.
TABle 1: Parameter of thermal decay curves of free radicals in fibroin at various states.

\begin{tabular}{lccc}
\hline Sample & $T_{\mathrm{d}} 1(\mathrm{~K})^{*}$ & $T_{\mathrm{d}} 2(\mathrm{~K})$ & $T_{\mathrm{d}} 3(\mathrm{~K})$ \\
\hline Dry fibroin & & 202.2 & 363.6 \\
Fibroin in water & & 209.4 & 279.5 \\
Fibroin in 40\% glycerol & 136.7 & 200.4 & 277.6 \\
Fibroin in 50\% glycerol & 132.5 & 208.3 & 281.0 \\
Average data & & $\mathbf{2 0 5 . 1 \pm 4 . 4}$ & $\mathbf{2 7 9 . 4 \pm 1 . 7 ^ { * * }}$ \\
\hline
\end{tabular}

${ }^{*}$ Transition temperature of activated dynamics; ${ }^{* *}$ excluding value for dry fibroin.

Most likely, in thermal activation, some of the cage-trapped radical pairs escape giving a rise of EPR spectra (Figures 1 and 3 ). In pure water, crystalline ice formation prevents the formation of the cages capable of stabilizing the free radical pairs.

Other than the initial increase in a total number of the spins, all free radical decay curves display two distinct regions that roughly can be divided into the temperature intervals of $77 \mathrm{~K}-250 \mathrm{~K}$ and $250 \mathrm{~K}-450 \mathrm{~K}$ (Figure 3(a)). The first derivatives of the decay curves are used to determine the transition temperatures, maxima of decay rates (Figure 3(b), Table 1). Interestingly, the transition temperature of $205.1 \pm$ $4.4 \mathrm{~K}$ (average for all) is about the same for all samples. Hydration of the protein does not shift significantly the transition temperature. However, in dry fibroin, the decay of free radicals responsible for the transition temperature of $\sim 205 \mathrm{~K}$ occurs in the much broader temperature interval (Figure 3). It indicates that, in the dry fibroin, the free radicals have more heterogeneous environments, leading to a broader distribution of activation energies. Besides that, the amplitude of the free radical decay in the first region is smaller compared to that of the hydrated samples. Free radical decays in the fibroin samples immersed in water and water/glycerol mixtures show the identical transition temperatures, $\sim 279.4 \mathrm{~K}$ (Figure 3, Table 1). However, fibroin in water shows much steeper transition around the temperature of $279.4 \mathrm{~K}$ compared to fibroin hydrated with glycerol mixtures.

Thus, the free radicals in fibroin hydrated with pure water have much more homogeneous environments, indicating more narrow distribution of activation energies. In dry fibroin, the transition at $\sim 279.4 \mathrm{~K}$ is absent. Instead, the second transition emerges at the temperature about $363.6 \mathrm{~K}$ shifted from $84 \mathrm{~K}$ toward higher temperature. Data indicate that protein dynamics responsible for the free radical decay with transition points of $279.4 \mathrm{~K}$ is strongly water-coupled.

The question arises if the nature of the free radicals in the first and second transition regions is identical. Difference EPR spectra of the free radicals of fibroin in the dry and hydrated forms were taken to identify the nature of free radicals decayed in both transition temperature regions (Figure 4).

The difference spectra in the first transition region are informative (Figure 4(a)). In both dry and hydrated samples, the quintet spectra with amplitude ratios about $1: 6: 20: 6: 1$ are obvious. Data indicate that the nature of the decayed radicals is the same for the dry and hydrated fibroin samples. The spectra can be assigned to the chain-end radicals for Gly 


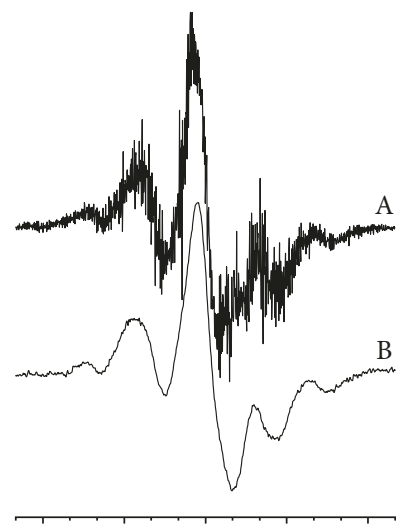

$\begin{array}{lllll}3300 & 3330 & 3360 & 3390 & 3420\end{array}$

(a)

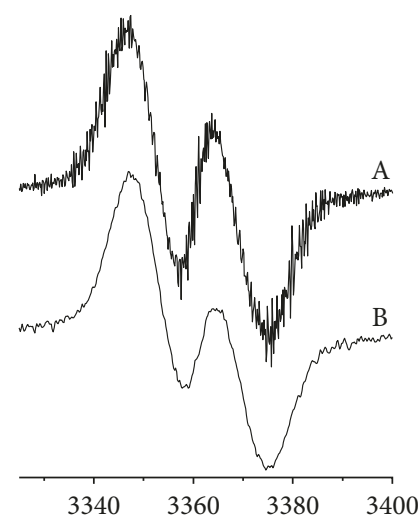

(b)
FIGURE 4: Difference EPR spectra of free radicals induced by UV irradiation of fibroin in weakly $(77-250 \mathrm{~K})$ and strongly watercoupled region (250-450). (a) A: water $(77 \mathrm{~K}-220 \mathrm{~K})$ and B: dry $(77 \mathrm{~K}-250 \mathrm{~K})$ forms. (b) A: fibroin in water $(280 \mathrm{~K}-290 \mathrm{~K})$ and B: dry fibroin $(380 \mathrm{~K}-400 \mathrm{~K})$.

and Ala residues, which are most abundant in fibroin. EPR spectra of the chain-end radicals of Gly $\left(-\mathrm{CONH}-\dot{\mathrm{C}} \mathrm{H}_{2}\right)$ and Ala (-CONH- $\dot{\mathrm{CH}}\left(\mathrm{CH}_{3}\right)$ ) show amplitude ratios of $1: 2: 1$ and $1: 4: 6: 4: 1$, respectively, arising from interactions with 2 and 4 equivalent protons, respectively. Disproportional contributions of the chain-end radicals of Gly, Ala, and other amino acids are responsible for the observed ratios of amplitudes in the difference spectra. In any case, the dominant contributions from Gly and Ala are apparent. Thus, under UV irradiation of fibroin along with $\alpha$-carbon radicals, the amino acid-specific free radicals localized at the chain end can be identified. The chain-end radicals show higher mobility but weakly coupled to water molecules. The bulk solvent in water, as well as glycerol solutions, is frozen at $205 \mathrm{~K}$. The frozen bulk solvent confines local dynamics (low amplitude) of the chainend groups and surrounding hydration shell.

In the second temperature transition region, the difference spectra of free radicals in fibroin in the dry and hydrated states (Figure 4(b)) indicate that types of free radicals are different compared with those of the first transition region. In both cases, well-resolved doublet spectra are evident. As it was mentioned above, these spectra can be assigned to the carbon radicals, $-\dot{\mathrm{C}}(\mathrm{OH})-\mathrm{NH}-$. Consistent with Figure 2, in the difference spectra, the doublet splitting in the hydrated sample is about $1 \mathrm{G}$ greater compared to that in the dry sample. In the second transition temperature $(279 \mathrm{~K})$, liquid bulk solvent permits relatively large-scale motion of the backbone of the protein that requires translational motion of solvent molecules. As can be expected, this motion is strongly water-coupled.

\section{Conclusions}

Thermal decay of free radicals induced by UV irradiation of silk fibroin in the dry and various hydrated forms revealed different dynamic modes of the protein. Each dynamic mode of the protein is characterized by the transition temperature.
Lowest transition temperature, $\sim 135 \mathrm{~K}$, observed only for fibroin hydrated in $40 \%$ and $50 \%$ glycerol solution characterizes escape of paired free radicals from the "cage." The transition temperature of $\sim 205 \mathrm{~K}$ was observed for all samples including the dry fibroin. Hydration of the protein significantly changed the magnitude of transition keeping transition temperature constant. Therefore, dynamic mode the protein corresponding to the transition temperature of $\sim 205 \mathrm{~K}$ is considered as weakly water-coupled. The transition temperature of $\sim 279 \mathrm{~K}$ was observed for hydrated samples, which is absent in the dry protein. Fibroin immersed in pure water shows the sharpest transition. The results indicate that dynamic mode of the protein characterized by transition temperature of $\sim 279 \mathrm{~K}$ is strongly water-coupled. In dry fibroin, the transition temperature of this dynamic mode observed at $\sim 364 \mathrm{~K}$ strongly shifted toward higher temperature. Thus, the thermal decay of free radicals induced by ultraviolet irradiation of fibroin discriminates the water-coupled dynamic modes of the protein. Nature of free radicals participating in the two distinct dynamic modes is different. However, the most likely difference in the temperature transition points is primarily related not to the nature of the radical intrinsically, but to the differences in the dynamics of the chain end and the chain middle regions. Future investigation is needed to justify this suggestion.

\section{Data Availability}

The data used to support the findings of this study are available from the corresponding author upon request.

\section{Conflicts of Interest}

The authors declare that they have no conflicts of interest.

\section{Acknowledgments}

The authors thank the Innovation Sector of the Institute of Physics of ANAS for providing access to the Bruker ELEXSYS E580 spectrometer and would like to thank Azerbaijan Academy of Sciences for financial support to conduct this study.

\section{References}

[1] L. Meinhold, J. C. Smith, A. Kitao, and A. H. Zewail, "Picosecond fluctuating protein energy landscape mapped by pressure temperature molecular dynamics simulation," Proceedings of the National Academy of Sciences, vol. 104, no. 44, pp. 17261-17265, 2007.

[2] H. Frauenfelder, "Physical concepts in biology-past and future," Physical Biology, vol. 14, no. 1, p. 010402, 2017.

[3] H. Frauenfelder, R. D. Young, and P. W. Fenimore, "Dynamics and the free-energy landscape of proteins, explored with the Mossbauer effect and quasi-elastic neutron scattering," Journal of Physical Chemistry B, vol. 117, no. 42, pp. 13301-13307, 2013.

[4] O. I. Aruoma, "Free radicals, oxidative stress, and antioxidants in human health and disease," Journal of the American Oil Chemists' Society, vol. 75, no. 2, pp. 199-212, 1998. 
[5] Q. Dong, H. Su, and D. Zhang, "In situ depositing silver nanoclusters on silk fibroin fibers supports by a novel biotemplate redox technique at room temperature," Journal of Physical Chemistry B, vol. 109, no. 37, pp. 17429-17434, 2005.

[6] B. D. Lawrence, J. K. Marchant, M. A. Pindrus, F. G. Omenetto, and D. L. Kaplan, "Silk film biomaterials for cornea tissue engineering," Biomaterials, vol. 30, no. 7, pp. 1299-1308, 2009.

[7] G. Yin, Z. Huang, M. Deng, J. Zeng, and J. Gu, "Preparation and cell response of bio-mineralized $\mathrm{Fe}(3) \mathrm{O}(4)$ nanoparticles," Journal of Colloid and Interface Science, vol. 363, no. 1, pp. 393-402, 2011.

[8] X.-X. Feng, L.-L. Zhang, J.-Y. Chen, Y.-H. Guo, H.-P. Zhang, and C.-I. Jia, "Preparation and characterization of novel nanocomposite films formed from silk fibroin and nano$\mathrm{TiO}_{2}$," International Journal of Biological Macromolecules, vol. 40, no. 2, pp. 105-111, 2007.

[9] Y. Tamada, "Sulfation of silk fibroin by chlorosulfonic acid and the anticoagulant activity," Biomaterials, vol. 25, no. 3, pp. 377-383, 2004.

[10] X. Wang, J. a. Kluge, G. G. Leisk, and D. L. Kaplan, "Sonication-induced gelation of silk fibroin for cell encapsulation," Biomaterials, vol. 29, no. 8, pp. 1054-1064, 2008.

[11] E. Wenk, A. J. Wandrey, H. P. Merkle, and L. Meinel, "Silk fibroin spheres as a platform for controlled drug delivery," Journal of Controlled Release, vol. 132, no. 1, pp. 26-34, 2008.

[12] A. Matsumoto, J. Chen, A. L. Collette et al., "Mechanisms of silk fibroin sol-gel transitions," Journal of Physical Chemistry B, vol. 110, no. 43, pp. 21630-21638, 2006.

[13] G. H. Altman, F. Diaz, C. Jakuba et al., "Silk-based biomaterials," Biomaterials, vol. 24, no. 3, pp. 401-416, 2003.

[14] C.-Z. Zhou, "Fine organization of Bombyx mori fibroin heavy chain gene," Nucleic Acids Research, vol. 28, no. 12, pp. 2413-2419, 2000.

[15] T. Lefèvre, M.-E. Rousseau, and M. Pézolet, "Protein secondary structure and orientation in silk as revealed by Raman spectromicroscopy," Biophysical Journal, vol. 92, no. 8, pp. 2885-2895, 2007.

[16] J. Shao, C. M. Carr, C. P. Rowlands, and J. Walton, "XPS, SIMS, and ESR Studies of UV/Ozone-irradiated silk and wool," Journal of the Textile Institute, vol. 90, no. 4, pp. 459-468, 1999.

[17] S. V. Mamedov, B. Akta, M. Cantürk et al., "The ESR signals in silk fibroin and wool keratin under both the effect of UVirradiation and without any external effects and the formation of free radicals," Biomaterials, vol. 23, no. 16, pp. 3405-3412, 2002.

[18] R. Liu, L. Xie, and K. Sheng, "ESR signals from silk fabrics irradiated by UV-rays," Nuclear Science and Techniques, vol. 18, no. 5, pp. 268-271, 2007.

[19] A. Sionkowska and A. Planecka, "The influence of UV radiation on silk fibroin," Polymer Degradation and Stability, vol. 96, no. 4, pp. 523-528, 2011.

[20] K. Setoyama, "ESR studies on free radicals in U.V.-irradiated silk fibroin," Journal of Sericultural Science of Japan, vol. 51, no. 4, pp. 271-278, 1982.

[21] D. N. Rockwood, R. C. Preda, T. Yucel, X. Wang, M. L. Lovett, and D. L. Kaplan, "Materials fabrication from Bombyx mori silk fibroin,” Nature Protocols, vol. 6, no. 10, pp. 1612-1631, 2011.

[22] K. M. Lvov and Y. A. Kim, "The ESR determination of protein a-helicity," Biopolymers, vol. 14, no. 1, pp. 83-91, 1975.

[23] M. T. Neves-Petersen, S. Klitgaard, T. Pascher et al., "Flash photolysis of cutinase: Identification and decay kinetics of transient intermediates formed upon UV excitation of aromatic residues," Biophysical Journal, vol. 97, no. 1, pp. 211-226, 2009.

[24] B. P. Partlow, M. Bagheri, J. L. Harden, and D. L. Kaplan, "Tyrosine templating in the self-assembly and crystallization of silk fibroin," Biomacromolecules, vol. 17, no. 11, pp. 3570-3579, 2016.

[25] P. S. Sherin, O. A. Snytnikova, and Y. P. Tsentalovich, "Tryptophan photoionization from prefluorescent and fluorescent states," Chemical Physics Letters, vol. 391, no. 1-3, pp. 44-49, 2004.

[26] J. Liu, J. Shao, and J. Zheng, "Radiation grafting/crosslinking of silk using electron-beam irradiation," Journal of Applied Polymer Science, vol. 91, no. 3, pp. 2028-2034, 2004.

[27] W. Li, X. Qiao, K. Sun, and X. Chen, "Effect of electron beam irradiation on the silk fibroin fiber/poly( $\varepsilon$-caprolactone) composite," Journal of Applied Polymer Science, vol. 113, no. 2, pp. 1063-1069, 2009. 

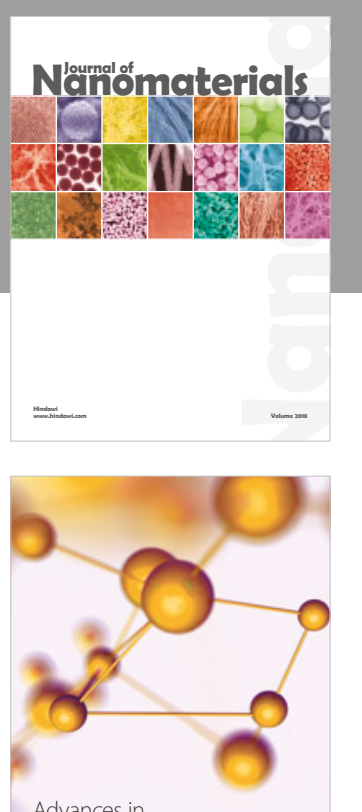

Physical Chemistry
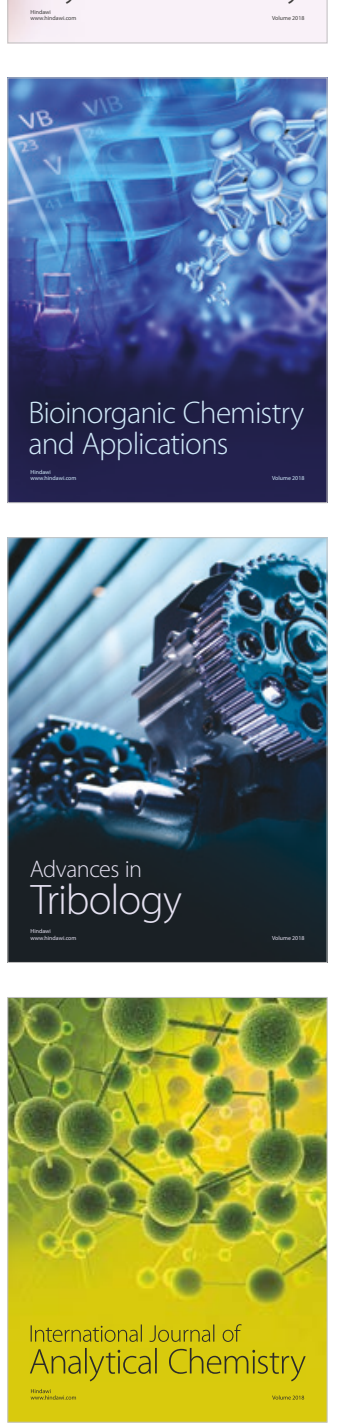

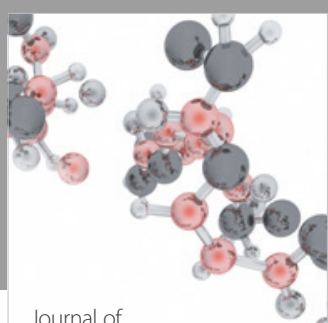

Analytical Methods

in Chemistry

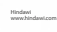

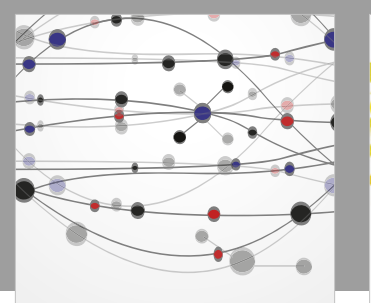

The Scientific World Journal

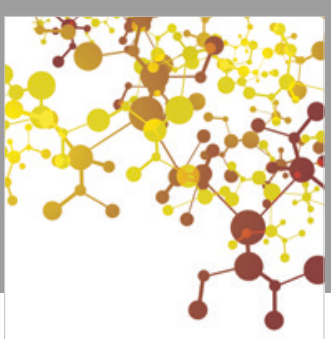

Journal of

Applied Chemistry
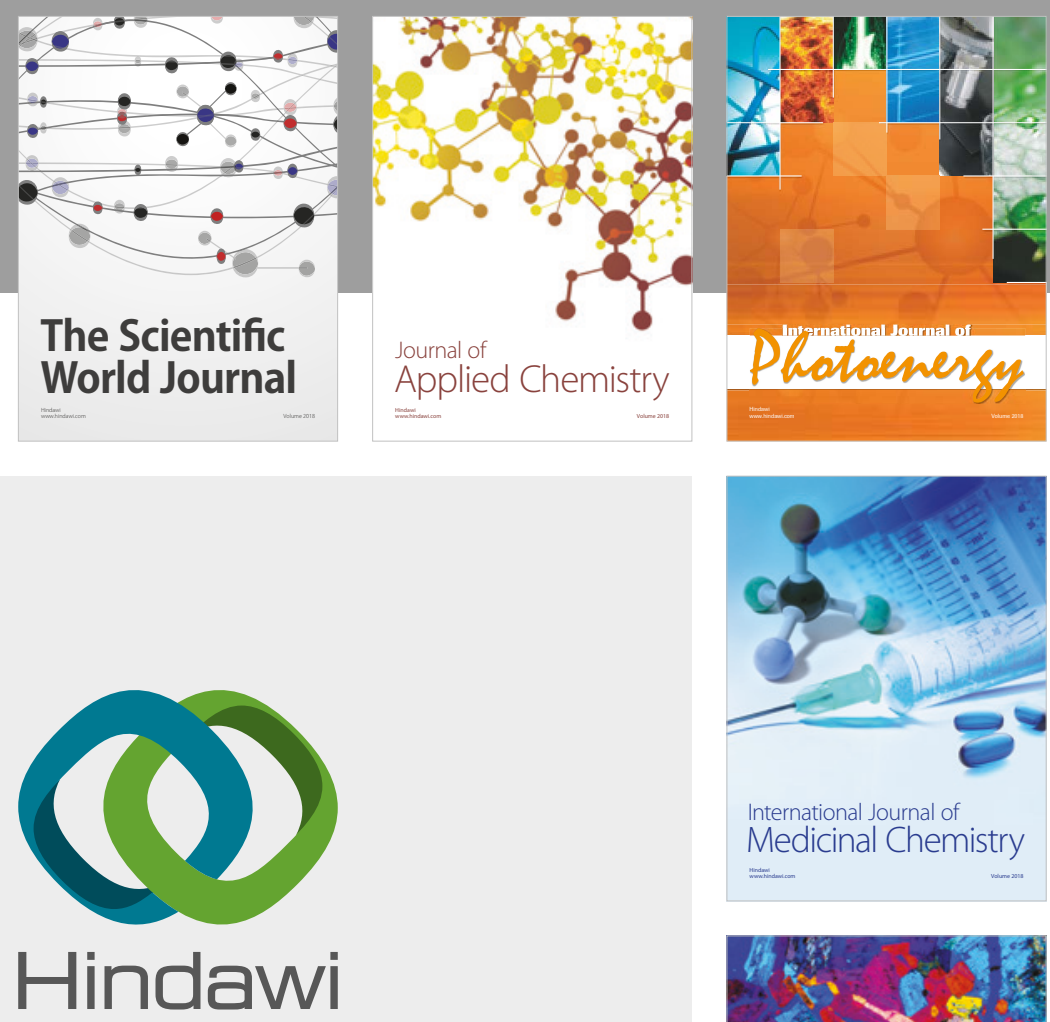

Submit your manuscripts at

www.hindawi.com
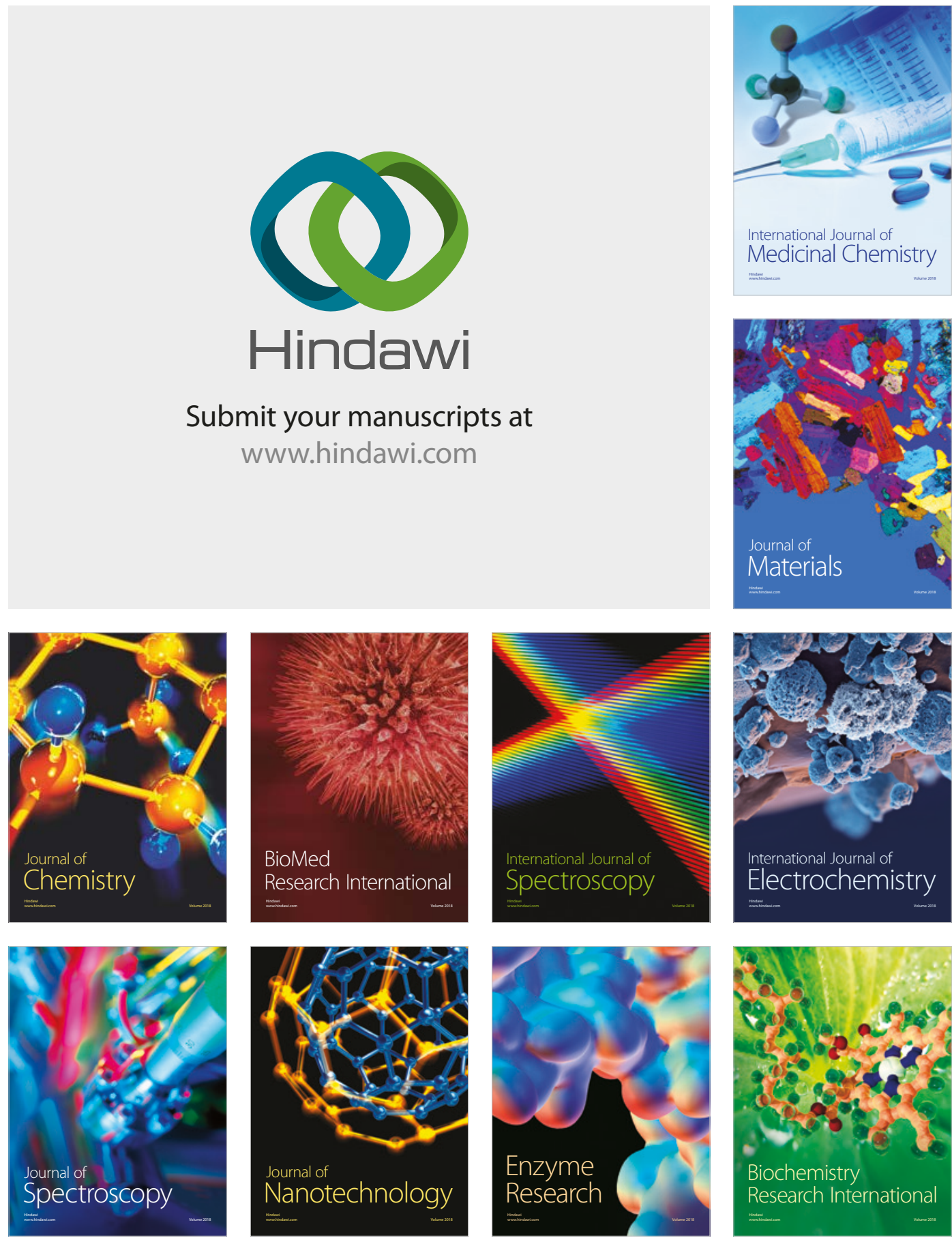
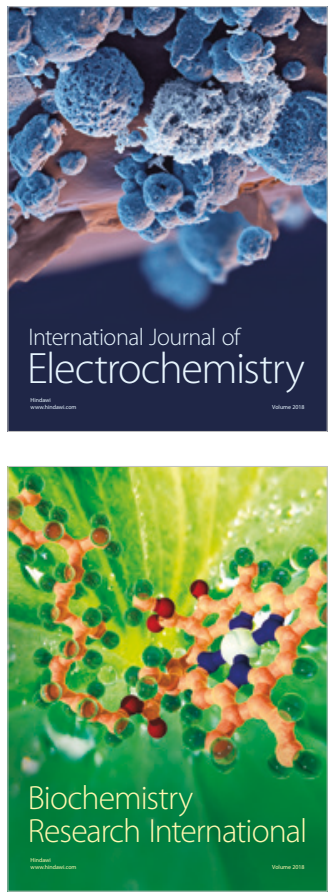